Supplement of Solid Earth, 8, 1211-1239, 2017

https://doi.org/10.5194/se-8-1211-2017-supplement

(C) Author(s) 2017. This work is distributed under

the Creative Commons Attribution 3.0 License.

(c) (1)

Supplement of

Constraints on the rheology of the lower crust in a strike-slip plate boundary: evidence from the San Quintín xenoliths, Baja California, Mexico

T. van der Werf et al.

Correspondence to: Vasileios Chatzaras (v.chatzaras@uu.nl)

The copyright of individual parts of the supplement might differ from the CC BY 3.0 License. 
Table S1. Estimated absorbance for principal vibrational directions based on Johnson and Rossman (2003)

\begin{tabular}{lcccc}
\hline \multirow{2}{*}{ Sample } & \multicolumn{3}{c}{ Oriented band areas $\left(\mathrm{cm}^{-2}\right)$} & \multirow{2}{*}{ Total band area $\left(\mathrm{cm}^{-2}\right)$} \\
\cline { 2 - 4 } & $\mathrm{x}$ & $\mathrm{y}$ & $\mathrm{z}$ & \\
\hline Andesine GRR1389 & 2455 & 1064 & 596 & 4115 \\
Oligioclase GRR580 & 999 & 394 & 117 & 1511 \\
Oligoclase GRR1280 & 2356 & 1310 & 215 & 3880 \\
Sanidine GRR638 & 753 & 269 & 542 & 1564 \\
Anorthite GRR1968 & 120 & 944 & 175 & 1239 \\
Microcline GRR1281 & 10917 & 2485 & 5986 & 19389 \\
Microcline GRR968 & 6892 & 1983 & 4704 & 13579 \\
\hline
\end{tabular}

$\mathrm{x}, \mathrm{y}, \mathrm{z}$ : principal vibrational directions 
Table S2. Representative mineral compositions

\begin{tabular}{|c|c|c|c|c|c|c|c|}
\hline SAMPLE & SQ-16 & SQW-75 & SQW-110 & SQW-114 & SQW-115 & SQW-76 & SQL-48 \\
\hline \multicolumn{8}{|l|}{ OLIVINE } \\
\hline \multicolumn{8}{|l|}{ wt. \% } \\
\hline $\mathrm{SiO}_{2}$ & 40.14 & 38.02 & 33.19 & 38.51 & 36.98 & & 36.56 \\
\hline $\mathrm{FeO}$ & 14.89 & 19.61 & 47.78 & 17.82 & 28.38 & & 29.82 \\
\hline $\mathrm{Fe}_{2} \mathrm{O}_{3}$ & 0.000 & 0.56 & 0.33 & 1.22 & 0.33 & & 0.00 \\
\hline $\mathrm{MnO}$ & 0.18 & 0.30 & 1.05 & 0.25 & 0.48 & & 0.40 \\
\hline MgO & 44.85 & 40.04 & 16.92 & 41.64 & 33.33 & & 31.66 \\
\hline $\mathrm{CaO}$ & 0.22 & 0.25 & 0.40 & 0.26 & 0.27 & & 0.53 \\
\hline Sum & 100.28 & 98.78 & 99.66 & 99.70 & 99.78 & & 98.96 \\
\hline \multicolumn{8}{|c|}{ cations per 4 oxygen atoms } \\
\hline $\mathrm{Si}$ & 1.00 & 0.99 & 0.99 & 0.99 & 1.00 & & 0.99 \\
\hline $\mathrm{Fe}^{2+}$ & 0.31 & 0.42 & 1.20 & 0.38 & 0.64 & & 0.68 \\
\hline $\mathrm{Fe}^{3+}$ & 0.00 & 0.02 & 0.01 & 0.02 & 0.01 & & 0.00 \\
\hline $\mathrm{Mn}$ & 0.00 & 0.01 & 0.03 & 0.01 & 0.01 & & 0.01 \\
\hline $\mathrm{Mg}$ & 1.67 & 1.55 & 0.76 & 1.59 & 1.34 & & 1.28 \\
\hline $\mathrm{Ca}$ & 0.01 & 0.01 & 0.01 & 0.08 & 0.01 & & 0.02 \\
\hline Sum & 2.99 & 3.00 & 2.99 & 3.00 & 3.00 & & 2.97 \\
\hline $\mathrm{X}_{\mathrm{Mg}}$ & 0.84 & 0.78 & 0.39 & 0.80 & 0.67 & & 0.65 \\
\hline \multicolumn{8}{|c|}{ ORTHOPYROXENE } \\
\hline \multicolumn{8}{|l|}{ wt. \% } \\
\hline $\mathrm{SiO}_{2}$ & 52.57 & 52.53 & 50.31 & 55.75 & 51.71 & 50.33 & 51.41 \\
\hline $\mathrm{TiO}_{2}$ & 0.03 & 0.08 & 0.10 & 0.03 & 0.15 & 0.12 & 0.08 \\
\hline $\mathrm{Al}_{2} \mathrm{O}_{3}$ & 3.39 & 4.01 & 2.27 & 1.31 & 3.49 & 2.22 & 2.93 \\
\hline $\mathrm{FeO}$ & 14.59 & 15.01 & 26.42 & 10.95 & 17.64 & 26.15 & 20.27 \\
\hline $\mathrm{Fe}_{2} \mathrm{O}_{3}$ & 1.11 & 0.78 & 1.78 & 0.40 & 1.56 & 1.11 & 2.22 \\
\hline $\mathrm{MnO}$ & 0.36 & 0.32 & 0.79 & 0.24 & 0.38 & 2.11 & 0.48 \\
\hline MgO & 26.49 & 26.19 & 17.74 & 29.31 & 24.01 & 17.26 & 22.35 \\
\hline $\mathrm{CaO}$ & 0.61 & 0.81 & 1.01 & 2.16 & 0.82 & 1.03 & 0.55 \\
\hline $\mathrm{Na}_{2} \mathrm{O}$ & 0.01 & 0.02 & 0.03 & 0.04 & 0.03 & 0.06 & 0.01 \\
\hline $\begin{array}{l}\text { Sum } \\
\text { cations pe }\end{array}$ & 99.16 & 99.73 & 100.44 & 100.20 & 99.79 & 100.40 & 100.29 \\
\hline $\mathrm{Si}$ & 0.95 & 0.95 & 0.96 & 0.96 & 0.99 & 0.95 & 0.95 \\
\hline $\mathrm{Ti}$ & 0.00 & 0.00 & 0.00 & 0.00 & 0.00 & 0.00 & 0.00 \\
\hline $\mathrm{Al}$ & 0.07 & 0.09 & 0.05 & 0.05 & 0.03 & 0.08 & 0.06 \\
\hline $\mathrm{Fe}^{2+}$ & 0.22 & 0.23 & 0.42 & 0.42 & 0.16 & 0.27 & 0.31 \\
\hline $\mathrm{Fe}^{3+}$ & 0.02 & 0.01 & 0.02 & 0.03 & 0.01 & 0.02 & 0.03 \\
\hline $\mathrm{Mn}$ & 0.01 & 0.01 & 0.03 & 0.01 & 0.00 & 0.01 & 0.01 \\
\hline $\mathrm{Mg}$ & 0.72 & 0.71 & 0.49 & 0.51 & 0.77 & 0.66 & 0.62 \\
\hline $\mathrm{Ca}$ & 0.01 & 0.02 & 0.02 & 0.02 & 0.04 & 0.02 & 0.01 \\
\hline $\mathrm{Na}$ & 0.00 & 0.00 & 0.00 & 0.00 & 0.00 & 0.00 & 0.00 \\
\hline Sum & 2.00 & 2.00 & 2.00 & 2.00 & 2.00 & 2.00 & 2.00 \\
\hline$X_{M g}$ & 0.75 & 0.75 & 0.53 & 0.53 & 0.82 & 0.69 & 0.64 \\
\hline
\end{tabular}


Table S2. Continued

\begin{tabular}{|c|c|c|c|c|c|c|c|}
\hline SAMPLE & SQ-16 & SQW-75 & SQW-110 & SQW-114 & SQW-115 & SQW-76 & SQL-48 \\
\hline \multicolumn{8}{|c|}{ CLINOPYROXENE } \\
\hline \multicolumn{8}{|l|}{$w t . \%$} \\
\hline $\mathrm{SiO}_{2}$ & 48.92 & 51.23 & 50.50 & 52.83 & 49.74 & & 49.93 \\
\hline $\mathrm{TiO}_{2}$ & 0.83 & 0.29 & 0.36 & 0.06 & 0.62 & & 0.65 \\
\hline $\mathrm{Al}_{2} \mathrm{O}_{3}$ & 7.17 & 4.66 & 3.61 & 3.27 & 5.09 & & 4.92 \\
\hline $\mathrm{FeO}$ & 4.63 & 4.16 & 11.80 & 5.71 & 6.41 & & 6.44 \\
\hline $\mathrm{Fe}_{2} \mathrm{O}_{3}$ & 0.77 & 1.67 & 1.78 & 1.10 & 2.68 & & 2.44 \\
\hline $\mathrm{MnO}$ & 0.12 & 0.13 & 0.41 & 0.20 & 0.22 & & 0.23 \\
\hline $\mathrm{MgO}$ & 13.67 & 14.37 & 11.76 & 17.24 & 13.19 & & 12.88 \\
\hline $\mathrm{CaO}$ & 22.47 & 23.07 & 19.67 & 19.33 & 21.38 & & 22.38 \\
\hline $\mathrm{Na}_{2} \mathrm{O}$ & 0.28 & 0.44 & 0.52 & 0.38 & 0.54 & & 0.44 \\
\hline Sum & 98.86 & 100.01 & 100.41 & 100.12 & 99.86 & & 100.30 \\
\hline \multicolumn{8}{|c|}{ cations per 6 oxygen atoms } \\
\hline $\mathrm{Si}$ & 1.82 & 1.88 & 1.90 & 1.93 & 1.85 & & 1.86 \\
\hline $\mathrm{Ti}$ & 0.02 & 0.01 & 0.01 & 0.00 & 0.02 & & 0.02 \\
\hline $\mathrm{Al}$ & 0.31 & 0.20 & 0.16 & 0.14 & 0.22 & & 0.22 \\
\hline $\mathrm{Fe}^{2+}$ & 0.14 & 0.13 & 0.37 & 0.17 & 0.20 & & 0.20 \\
\hline $\mathrm{Fe}^{3+}$ & 0.02 & 0.05 & 0.05 & 0.03 & 0.08 & & 0.07 \\
\hline $\mathrm{Mn}$ & 0.00 & 0.00 & 0.01 & 0.01 & 0.01 & & 0.01 \\
\hline $\mathrm{Mg}$ & 0.76 & 0.79 & 0.66 & 0.94 & 0.73 & & 0.71 \\
\hline $\mathrm{Ca}$ & 0.89 & 0.91 & 0.79 & 0.76 & 0.85 & & 0.89 \\
\hline $\mathrm{Na}$ & 0.02 & 0.03 & 0.04 & 0.03 & 0.04 & & 0.03 \\
\hline Sum & 3.99 & 4.00 & 4.00 & 4.00 & 3.99 & & 4.00 \\
\hline $\mathrm{X}_{\mathrm{Mg}}$ & 0.82 & 0.82 & 0.61 & 0.82 & 0.73 & & 0.73 \\
\hline \multicolumn{8}{|l|}{ SPINEL } \\
\hline \multicolumn{8}{|l|}{$w t . \%$} \\
\hline $\mathrm{TiO}_{2}$ & 0.18 & 0.04 & & 0.02 & & & \\
\hline $\mathrm{Al}_{2} \mathrm{O}_{3}$ & 60.14 & 60.16 & & 61.38 & & & \\
\hline $\mathrm{Cr}_{2} \mathrm{O}_{3}$ & 1.13 & 0.03 & & 0.90 & & & \\
\hline $\mathrm{FeO}$ & 18.98 & 17.64 & & 14.72 & & & \\
\hline $\mathrm{Fe}_{2} \mathrm{O}_{3}$ & 4.44 & 6.11 & & 4.45 & & & \\
\hline $\mathrm{MnO}$ & 0.19 & 0.15 & & 0.09 & & & \\
\hline $\mathrm{MgO}$ & 14.96 & 15.41 & & 17.45 & & & \\
\hline $\mathrm{CaO}$ & 0.06 & 0.02 & & 0.04 & & & \\
\hline Sum & 100.08 & 99.56 & & 99.02 & & & \\
\hline \multicolumn{8}{|c|}{ cations per 4 oxygen atoms } \\
\hline $\mathrm{Ti}$ & 0.00 & 0.00 & & 0.00 & & & \\
\hline Al & 1.87 & 1.88 & & 1.89 & & & \\
\hline $\mathrm{Cr}$ & 0.02 & 0.00 & & 0.02 & & & \\
\hline $\mathrm{Fe}^{2+}$ & 0.42 & 0.39 & & 0.32 & & & \\
\hline $\mathrm{Fe}^{3+}$ & 0.09 & 0.12 & & 0.09 & & & \\
\hline $\mathrm{Mn}$ & 0.00 & 0.00 & & 0.00 & & & \\
\hline $\mathrm{Mg}$ & 0.59 & 0.61 & & 0.68 & & & \\
\hline $\mathrm{Ca}$ & 0.00 & 0.00 & & 0.00 & & & \\
\hline Sum & 3.00 & 3.00 & & 3.00 & & & \\
\hline$X_{M g}$ & 0.54 & 0.55 & & 0.62 & & & \\
\hline
\end{tabular}


Table S2. Continued

\begin{tabular}{|c|c|c|c|c|c|c|c|}
\hline SAMPLE & SQ-16 & SQW-75 & SQW-110 & SQW-114 & SQW-115 & SQW-76 & SQL-48 \\
\hline \multicolumn{8}{|c|}{ PLAGIOCLASE (Recrystallized grains) } \\
\hline \multicolumn{8}{|l|}{ wt. \% } \\
\hline $\mathrm{SiO}_{2}$ & 43.65 & 44.31 & & 44.31 & 46.23 & 60.08 & 44.58 \\
\hline $\mathrm{Al}_{2} \mathrm{O}_{3}$ & 36.32 & 34.96 & & 34.84 & 33.44 & 25.80 & 34.55 \\
\hline $\mathrm{FeO}$ & 0.00 & 0.00 & & 0.00 & 0.00 & 0.00 & 0.00 \\
\hline $\mathrm{Fe}_{2} \mathrm{O}_{3}$ & 0.00 & 0.00 & & 0.00 & 0.00 & 0.00 & 0.00 \\
\hline $\mathrm{MgO}$ & 0.00 & 0.01 & & 0.04 & 0.04 & 0.02 & 0.01 \\
\hline $\mathrm{CaO}$ & 19.92 & 19.01 & & 19.28 & 17.73 & 7.68 & 19.09 \\
\hline $\mathrm{Na}_{2} \mathrm{O}$ & 0.30 & 0.77 & & 0.74 & 1.53 & 7.02 & 0.79 \\
\hline $\mathrm{K}_{2} \mathrm{O}$ & 0.01 & 0.00 & & 0.00 & 0.03 & 0.31 & 0.05 \\
\hline Sum & 100.18 & 99.06 & & 99.21 & 99.00 & 100.91 & 99.08 \\
\hline \multicolumn{8}{|c|}{ cations per 8 oxygen atoms } \\
\hline Si & 2.02 & 2.07 & & 2.07 & 2.15 & 2.65 & 2.08 \\
\hline Al & 1.98 & 1.92 & & 1.91 & 1.83 & 1.34 & 1.90 \\
\hline $\mathrm{Fe}^{2+}$ & 0.00 & 0.00 & & 0.00 & 0.00 & 0.00 & 0.00 \\
\hline $\mathrm{Fe}^{3+}$ & 0.00 & 0.00 & & 0.00 & 0.00 & 0.00 & 0.00 \\
\hline $\mathrm{Mg}$ & 0.00 & 0.00 & & 0.00 & 0.00 & 0.00 & 0.00 \\
\hline $\mathrm{Ca}$ & 0.99 & 0.95 & & 0.96 & 0.88 & 0.36 & 0.95 \\
\hline $\mathrm{Na}$ & 0.03 & 0.07 & & 0.07 & 0.14 & 0.60 & 0.07 \\
\hline $\mathrm{K}_{2} \mathrm{O}$ & 0.00 & 0.00 & & 0.00 & 0.00 & 0.02 & 0.00 \\
\hline Sum & 5.01 & 5.01 & & 5.01 & 5.00 & 4.98 & 5.01 \\
\hline \multicolumn{8}{|c|}{ PLAGIOCLASE (Symplectites/melt) } \\
\hline \multicolumn{8}{|l|}{$w t . \%$} \\
\hline $\mathrm{SiO}_{2}$ & 46.95 & 47.51 & & 59.98 & 52.72 & & \\
\hline $\mathrm{Al}_{2} \mathrm{O}_{3}$ & 34.06 & 32.66 & & 15.32 & 28.66 & & \\
\hline $\mathrm{FeO}$ & 0.00 & 0.31 & & 2.15 & 0.00 & & \\
\hline $\mathrm{Fe}_{2} \mathrm{O}_{3}$ & 0.00 & 0.00 & & 3.64 & 0.00 & & \\
\hline $\mathrm{MgO}$ & 0.17 & 0.21 & & 3.21 & 0.16 & & \\
\hline $\mathrm{CaO}$ & 17.37 & 16.73 & & 6.39 & 12.43 & & \\
\hline $\mathrm{Na}_{2} \mathrm{O}$ & 1.62 & 1.90 & & 5.50 & 4.43 & & \\
\hline $\mathrm{K}_{2} \mathrm{O}$ & 0.01 & 0.02 & & 1.08 & 0.21 & & \\
\hline Sum & 100.18 & 99.33 & & 97.26 & 98.61 & & \\
\hline \multicolumn{8}{|c|}{ cations per 8 oxygen atoms } \\
\hline Si & 2.15 & 2.20 & & 2.80 & 2.42 & & \\
\hline $\mathrm{Al}$ & 1.84 & 1.78 & & 0.84 & 1.55 & & \\
\hline $\mathrm{Fe}^{2+}$ & 0.00 & 0.00 & & 0.08 & 0.00 & & \\
\hline $\mathrm{Fe}^{3+}$ & 0.00 & 0.01 & & 0.13 & 0.00 & & \\
\hline $\mathrm{Mg}$ & 0.01 & 0.01 & & 0.23 & 0.01 & & \\
\hline $\mathrm{Ca}$ & 0.85 & 0.83 & & 0.32 & 0.61 & & \\
\hline $\mathrm{Na}$ & 0.14 & 0.17 & & 0.50 & 0.40 & & \\
\hline $\mathrm{K}_{2} \mathrm{O}$ & 0.00 & 0.00 & & 0.06 & 0.01 & & \\
\hline Sum & 5.00 & 5.00 & & 4.97 & 5.01 & & \\
\hline
\end{tabular}


Table S3. The volume percentage, magnesium numbers, $\mathrm{Al}_{2} \mathrm{O}_{3}$ in orthopyroxene and clinopyroxene, $\mathrm{CaO}$ in plagioclase and clinopyroxene in samples SQ-16, SQW-75, and sQW-114.

\begin{tabular}{lccc}
\hline \multicolumn{1}{c}{ Xenolith } & SQ-16 & SQW-75 & SQW-114 \\
\hline Vol\% & & & \\
$\mathrm{Plg}$ & 43.8 & 50.1 & 15.9 \\
$\mathrm{Cpx}$ & 19.7 & 32.2 & 19.5 \\
$\mathrm{Sp}$ & 13.0 & 2.2 & 13.0 \\
$\mathrm{OI}$ & 22.5 & 2.0 & 23.7 \\
Opx & - & 14.0 & 27.9 \\
Total & 99.0 & 100.5 & 100.0 \\
\hline $\mathrm{X}_{\mathrm{Mg}}$ & & & \\
$\mathrm{Cpx}$ & 0.97 & 0.82 & 0.83 \\
$\mathrm{Sp}$ & 0.59 & 0.55 & 0.62 \\
$\mathrm{OI}$ & 0.82 & 0.75 & 0.80 \\
$\mathrm{Opx}$ & - & 0.75 & 0.82 \\
\hline $\mathrm{Al} \mathrm{I}_{3}$ & & & \\
$\mathrm{Cpx}$ & 0.16 & 0.10 & 0.07 \\
$\mathrm{Opx}$ & - & 0.04 & 0.01 \\
$\mathrm{CaO}$ & & & \\
$\mathrm{Plg}$ & 0.85 & 0.94 & 0.96 \\
$\mathrm{Cpx}$ & 0.89 & 0.91 & 0.75 \\
\hline
\end{tabular}

Opx is on a 3 oxygen basis

Table S4. Results of two-pyroxene geothermometry

\begin{tabular}{lcccc}
\hline \multirow{2}{*}{ Sample } & \multicolumn{4}{c}{$\mathrm{T}\left({ }^{\circ} \mathrm{C}\right)$ calculated at a pressure of 600 MPa } \\
\cline { 2 - 5 } & $2-\mathrm{Px} \mathrm{BK}^{\mathrm{a}}$ & $2-\mathrm{Px} \mathrm{T}^{\mathrm{b}}$ & 2-Px Avg & 1 std dev \\
\hline SQ-16 & 732 & 770 & 751 & 27 \\
SQL-48 & 739 & 790 & 765 & 36 \\
SQW-75 & 741 & 777 & 759 & 25 \\
SQW-110 & 824 & 947 & 886 & 87 \\
SQW-114 & 863 & 908 & 886 & 32 \\
SQW-115 & 834 & 874 & 854 & 28 \\
\hline
\end{tabular}

${ }^{\mathrm{a}}$ Brey and Köhler (1990); ${ }^{\mathrm{b}}$ Taylor (1998) 
Table S5. Experimental flow law parameters

\begin{tabular}{|c|c|c|c|c|c|c|c|}
\hline Lithology & $\begin{array}{l}\text { Deformation } \\
\text { Mechanism }\end{array}$ & $\mathrm{A}\left(\mathrm{MPa}^{-\mathrm{n}} \mu \mathrm{m}^{\mathrm{m}} \mathrm{s}^{-1}\right)$ & $\mathrm{n}$ & $\mathrm{m}$ & $\begin{array}{c}\mathrm{Q} \\
(\mathrm{kJ} / \mathrm{mol})\end{array}$ & $\mathrm{V}\left(\mathrm{m}^{3} / \mathrm{mol}\right)$ & Reference \\
\hline An100, Dry & Dis & $5.01 \times 10^{12}$ & 3 & 0 & 648 & & Rybacki \& Dresen (2000) \\
\hline An100, Dry & Dif & $1.26 \times 10^{12}$ & 1 & 3 & 467 & & Rybacki \& Dresen (2000) \\
\hline An100, Wet & Dis & $3.98 \times 10^{2}$ & 3 & 0 & 356 & & Rybacki \& Dresen (2000) \\
\hline An100, Wet & Dif & $5.01 \times 10^{1}$ & 1 & 3 & 170 & & Rybacki \& Dresen (2000) \\
\hline An25Di $_{45}$, Dry & Dis & $6.15 \times 10^{-4}$ & 3.03 & 0 & 701 & & Dimanov \& Dresen (2005) \\
\hline An25Di ${ }_{45}$, Dry & Dif & $1.26 \times 10^{6}$ & 1 & 3 & 454 & & Dimanov \& Dresen (2005) \\
\hline An25Di 35 , Wet & Dis & $5.25 \times 10^{-15}$ & 3.01 & 0 & 391 & & Dimanov \& Dresen (2005) \\
\hline An25Di ${ }_{35}$, Wet & Dif & $1.24 \times 10^{0}$ & 1 & 3 & 291 & & Dimanov \& Dresen (2005) \\
\hline Olivine, Dry & Dis & $1.1 \times 10^{5}$ & 3.5 & 0 & 530 & $1.8 \times 10^{-5}$ & Hirth \& Kohlstedt (2003) \\
\hline Olivine, Dry & Dif & $1.5 \times 10^{9}$ & 1 & 3 & 375 & $1 \times 10^{-6}$ & Hirth \& Kohlstedt (2003) \\
\hline Olivine, Dry & disGBS & $6.31 \times 10^{4}$ & 2.9 & 0.7 & 445 & & Hansen et al. (2011) \\
\hline
\end{tabular}

The preexponential parameters (A) in italics are recalculated based on this study (see text for explanation); Dis: Dislocation creep,

Dif: Diffusion creep, disGBS: Dislocation accommodated grain boundary sliding

\section{Table References}

Dimanov, A., and Dresen, G.: Rheology of synthetic anorthite - diopside aggregates: Implications for ductile shear zone, J. Geophys. Res-sol. Ea., 110(B7), 2005.

Hansen, L. N., Zimmerman, M. E., and Kohlstedt, D. L.: Grain boundary sliding in San Carlos olivine: Flow law parameters and crystallographic - preferred orientation, J. Geophys. Res-sol. Ea., 116(B8), 2011.

Hirth, G., and Kohlstedt, D.: Rheology of the upper mantle and the mantle wedge: A view from the experimentalists. Inside the subduction Factory, J. Eiler, American Geophysical Union, Washington, D.C., 83-105, 2003.

Rybacki, E., and Dresen, G.: Dislocation and diffusion creep of synthetic anorthite aggregates, J. Geophys. Res-Sol. Ea., 105(B11), 26017-26036, 2000. 